\title{
Steering Angle Prediction Based on Road Direction using Convolution Neural Network (CNN)
}

\author{
Mr. Aires Da Conceicao ${ }^{1}$, Dr. Sheshang Degadwala ${ }^{2}$ \\ ${ }^{1}$ U.G. Student, Sigma Institute of Engineering, Vadodara, Gujarat, India \\ ${ }^{2}$ Associate Professor, Sigma Institute of Engineering, Vadodara, Gujarat, India
}

\author{
Article Info \\ Volume 7, Issue 4 \\ Page Number: 88-95 \\ Publication Issue : \\ July-August-2020
}

\section{Article History}

Accepted : 20 July 2020

Published : 25 July 2020

\section{ABSTRACT}

Self driving vehicle is a vehicle that can drive by itself it means without human interaction. This system shows how the computer can learn and the over the art of driving using machine learning techniques. Therefore for a car achieving the autonomous ability it must show the control of human activities while driving. Those activities include control of steering wheel. There exist different techniques to control the steering angle and one of them is CNN. In this article is going to show how CNN can be used to predict the steering angle.

Keywords :- Self-driving car, CNN, Steering angle, Prediction.

\section{INTRODUCTION}

Nowadays the world is moving so fast concern about the new technologies. The automotive industry is also one of the most affect by this upcoming technology. Self driving vehicle is an example of that. This kind of vehicle is no longer an imagination nowadays it turned into a reality and may enhance the future systems where computers take over the art of driving. Autonomous car uses different kinds of technologies such as GPS to help with navigation and use sensors like RADAR or LIDAR that are used to avoid collisions. There are a huge interest in the development of autonomous vehicle in recent years. However one of the most important aspect to take in consideration while driving is controlling the steering angle. The autonomous car has the ability of predicting the steering angle of the car wheel based on the road direction. This ability can be achieved by different techniques one of them is CNN. CNN means Convolutional Neural Network it it a Deep Learning technique where fully layered neurons are connected together in order to perform the computation. The data to be passed to CNN need to preprocess for that some segmentation methods are applied.

\section{RELATED WORKS}

End to End Self-Driving Using Deep Neural Networks with Multi-auxiliary Tasks Dan Wang, Junjie Wen, Yuyong Wang, Xiangdong Huang and Feng Pei the article Networks with Multi-auxiliary Tasks they intend to compare various end-to-end multi-task deep learning networks by combining deep convolutional 
neural network along with long short-term memory recurrent neural network (CNN-LSTM), which could obtain visual spatial data and dynamic temporal values at same instant in the driving environment or scenarios, and giving a better steer or improve steering angle and speed predictions.

Controlling Steering Angle for Cooperative Selfdriving Vehicles utilizing CNN and LSTM-based Deep Networks by Rodolfo Valiente, Mahdi Zaman, Sedat Ozer and Yaser P. Fallah the research they proposed a soluction of the problem generated while predicting steering angles directly from the raw input images. When temporal library or dependencies among the image frames is ignored. They presented and studied a new deep architecture for predicting the steering angle automatically or autonomously by utilizing LSTM. The deep structure is a connected network that combined CNN, LSTM and fully connected layers. It utilizes both present images and future images as an input value to handle the steering angle.

Steering angle prediction in autonomous vehicle using deep learning by Vaibhav Singhal, Snehal Gugale, Rohit Agarwal, Pritam Dhake and Urmila Kalshetti the article show how deep learning can be effectively used for prediction of steering angle of the vehicle based on the road curvature. It uses data input from 3 different angles (left camera, center camera , right camera) those data are first preprocessed and then along with the other attributes such as speed of the vehicle are the fed into the CNN algorithm for generate the CNN model. The system works with a low dimension image the consequently reduces the training time.

\section{PROPOSED SYSTEM}

The proposed system is based on Nvidia architecture for convolutional neural network algorithm.
1) Input data

The input data of the system is the recording frame or image of the road captured from the camera that is plotted on top of the vehicle. Those set of frames will be fed into the system in order to make the required prediction of the road direction.

\section{2) System Algorithm}

This system uses supervised learning. It is a IA technique in which label data are used to feed the model in order to make prediction on the related data. The algorithm used in this system is convolutional neural network. CNN has been proving to be one of the best algorithm for prediction over a large amount of data.

At the first phase the image set is divided into 2 section which are training dataset and validation dataset. The training dataset is fed into the image enhancement algorithm to remove the noise that may be presented on the image and then random shift and rotation are applied on image for achieving the looking of moving vehicle. At second phase the preprocessed image is fed into the CNN computation after all if overfitting is encountered then the weight adjustment is made until functional model is achieved. At the last phase

The label image of the road gathered from camera is used to make the prediction of the road direction using the generated model.

\section{3) Output data}

Output data of the system will computed based on the similar pattern of the input data, all the convolution operation will be made by CNN algorithm. The output data of the system is the road direction at given moment based on the road image. 


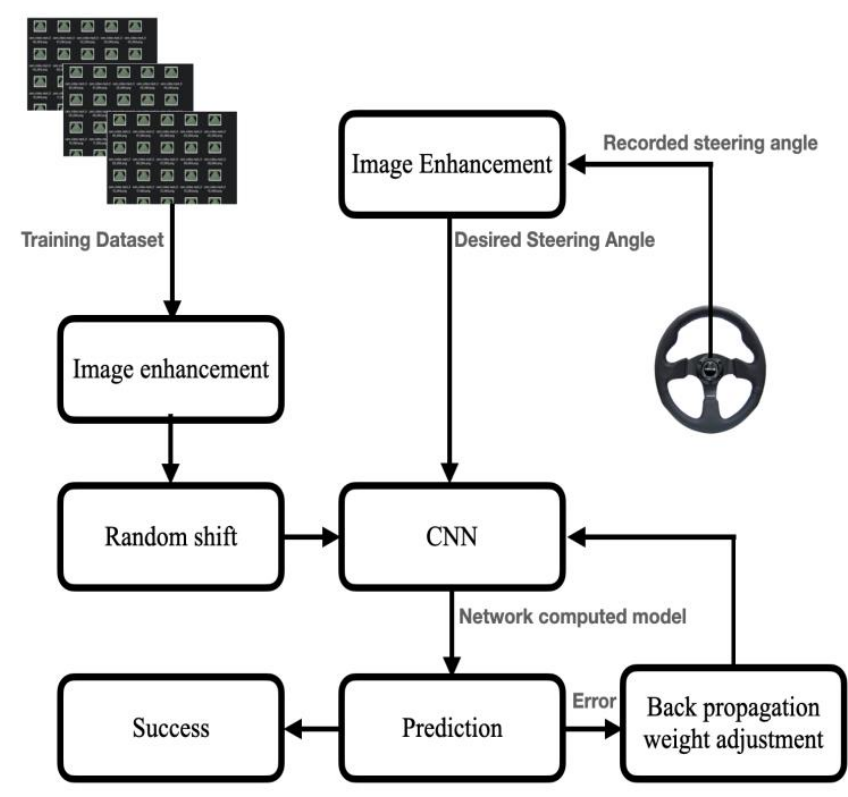

Fig 13: Proposed system

\section{BACKGROUND THEORY}

1) Gaussian Blur

A Gaussian blur technique is used to convolve with the image and effectively reduce the effects caused by the noise and. The equation for a Gaussian blur used is :

$$
\text { Hij }=\frac{1}{2 \pi \sigma^{2}} \exp \left(\frac{(1-(k+1))^{2}+(1+(k+1))^{2}}{2 \sigma^{2}}\right) ; 1 \leq \mathrm{i}, \mathrm{j} \leq
$$$$
(2 \mathrm{k}+1)
$$

\section{2) Histogram Equalization}

Histogram Equalization is a image processing technique used to improve the quality of the image. Image with good quality is an image with a good contrast. It is accomplished by spreading out the most intense values in the dynamic range of the image or stretching the most intense range of the image. Histogram equalization is used to gain a better image quality by increasing its contrast. This allows regions of the image with low contrast to get a high image contrast. Therefore histogram equalization is used to increase image quality, an image with good quality is an image with high contrast and it is achieved by spreading dynamic range of the image.
Formula:

$$
h(v)=\operatorname{round}\left(\frac{c d f(v)-c d f_{\min }}{(M X N)-c f_{\text {min }}} \times(L-1)\right)
$$

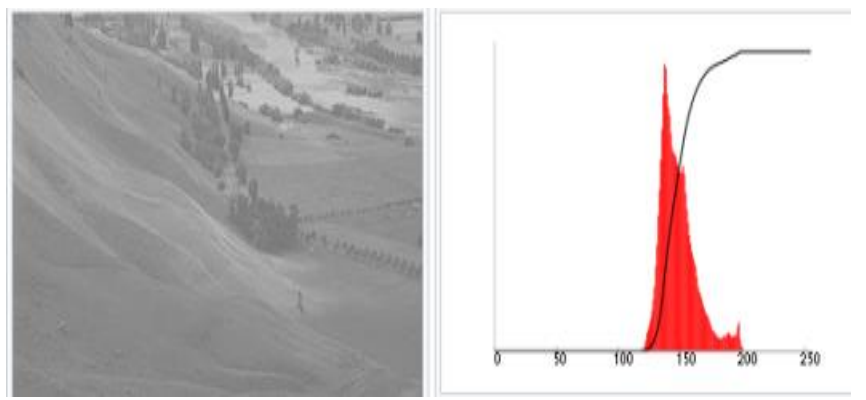

Fig. 1: Image before Histogram Equalization
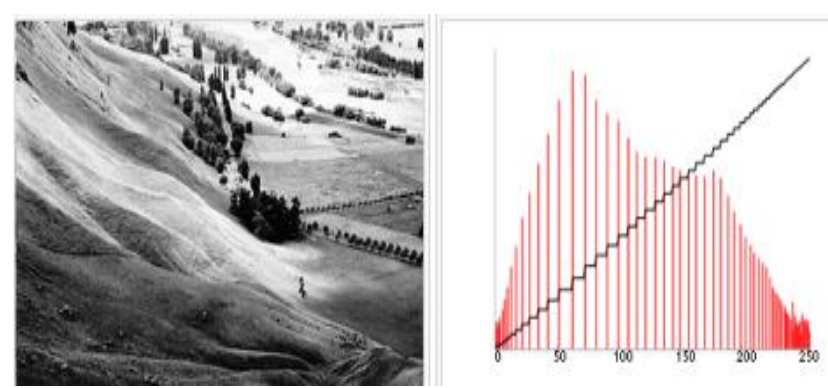

Fig. 2: Image after Histogram Equalization

\section{3) Feature Extraction}

Hough Transform is a feature extraction technique used to identify different types of shape based on voting occurrence. In this case is used to detect the lane line on the incoming frames from the camera. The line is detected by analyzing all points that are passed through the line and this points are represented as a sinusoidal waves passing through a common line as shown in the image below. The line formula is:

$$
\rho=x \cos (\theta)+x \sin (\theta)
$$

Were $\rho$ is the given length of the normal line and $\theta$ is the total angle between the normal line and the $\mathrm{x}$-axis. 

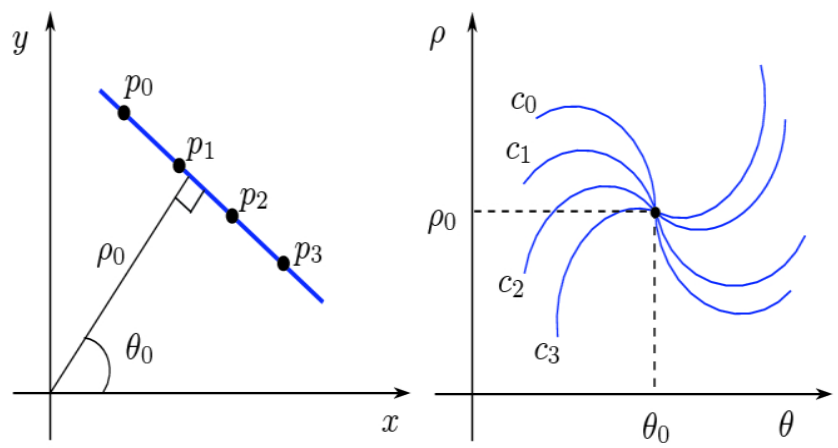

Fig 3: Hough Transform.

Feature extraction is a technique of reducing the dimensionality of the processed data for manage it better.
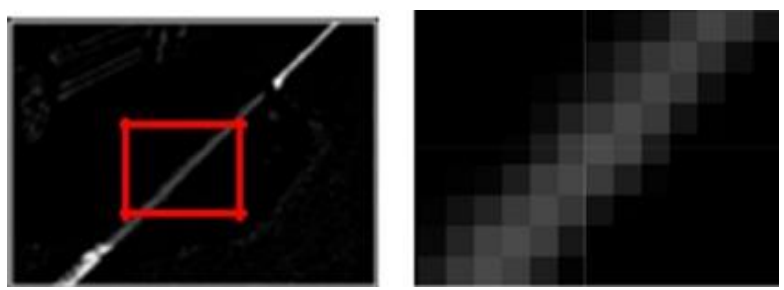

Fig 4: Feature Extraction

4) Epoch

It is used to determine the number of time all samples of training dataset are used once to update the weights. One epoch defines the opportunity to update the internal model parameters based on samples in the training dataset. It is a parameter that determine the number of time the learning program or algorithm will process through the whole training dataset in order to minimize the model error rate.

\section{5) Activation Function}

Activation function is responsible for activating or deactivate the neurons in order to get de desired output. It defines as neurons operation, the inputs of activation are the bias weights. Activation function is responsible to decide in any neural network if the receiving information is relevant or not. ELU means Exponential Linear Unit it is a function which converge values to zero much faster and generating more accurate results. The difference between ELU with most other functions is ELU has an additional alpha constant value which is positive. ELU is like RELU but ELU converges to negative inputs different from RELU. ELU performs smoothly until its output equal to - $\alpha$ whereas RELU performs sharply smooth.

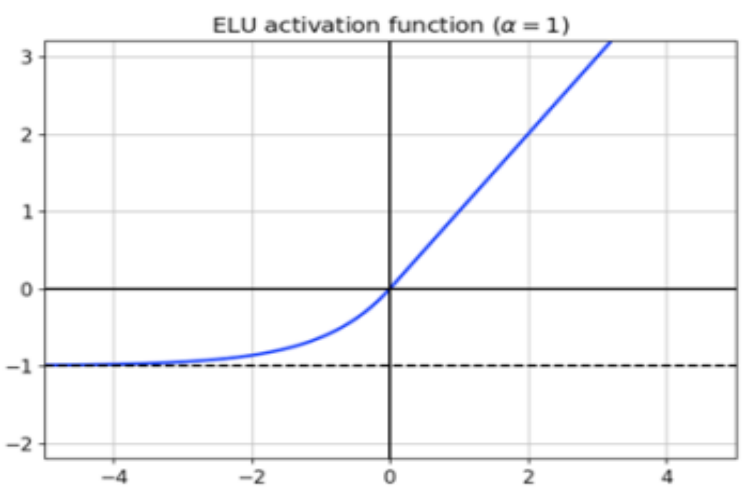

Fig.5: ELU Activation Function

\section{v. CNN COMPUTATION}

\section{1) Data Collection}

The first step for computing steering angle prediction is to get a stream of good quality image

of the road. More images leads to high accuracy but for this article 452 labeled images has been used. All the images must be of the same size and dimension. Based on Nvidia the image dimention should be (66, 200) where 66 represent the length and 200 represent the width.

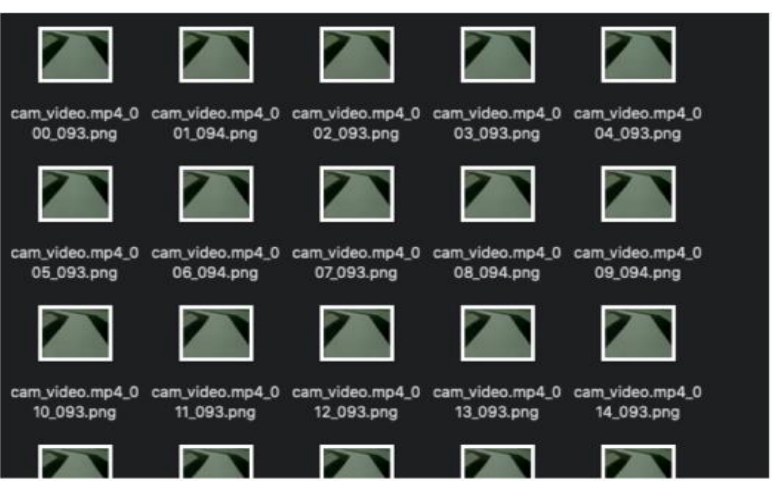

Fig.6: 452 labeled images data.

While collecting the images we have to make sure to collect the image in all direction of the road that include (Right, Center and Left). 


\section{2) Data Plot}

The below image shows the angles labeled in the images. All the images in our data set are labeled with the steering angle. While collecting the image the steering angle will be assigned at each frame based of the road direction it means the values less than 80 are considered left, values between 80 to 120 are considered center and values greater than 120 are considered Right.

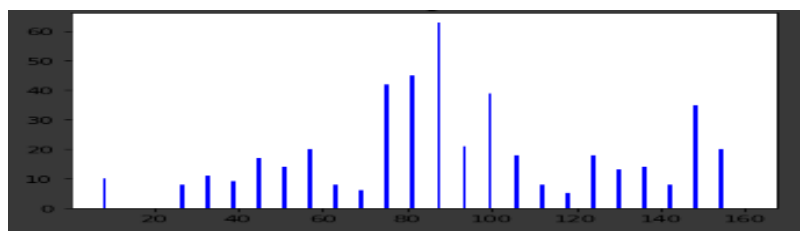

Fig.7: Labeled Images Representation Based on Steering Value.

\section{3) Histogram Equalization}

After collecting the image data some data may come with a bad contrast due to position of the light so to get a good image quality we have to use the histogram equalization. Histogram equalization is used to increase the image quality by spreading its dynamic range.

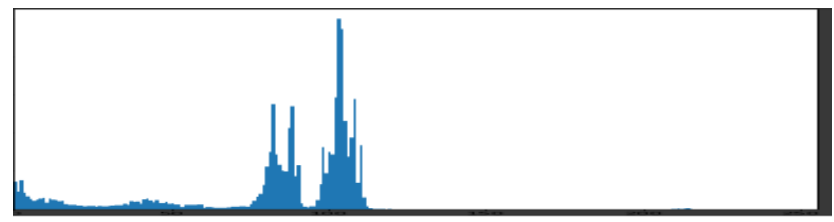

Fig.8: Histogram of Image

4) Trained and validation data

The collected data is converted into two dataset that are training dataset and validation dataset.

Training dataset is a collection of labeled images and the size of the training dataset is 452 label images. Training data will be passed into CNN convolution for generate our model and validation dataset is a collection of 113 images that will be used for testing the accuracy of the generated model.

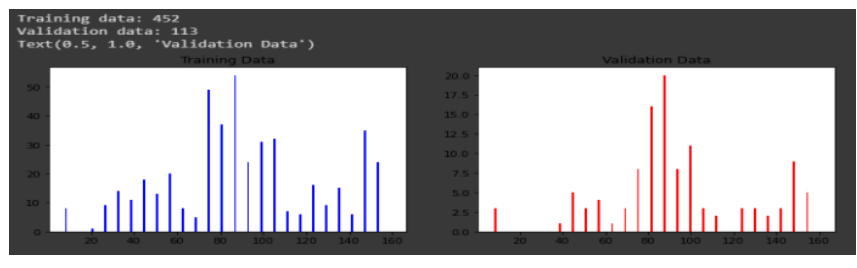

Fig.9: 452 training images and 113 validation images.

\section{5) YUV Image}

Based on Nvidia model the YUV Image has proven to be very efficient for image feature extraction so we have converted our data set into YUV image.

Now set the region of interest is settled that is the most interested region.

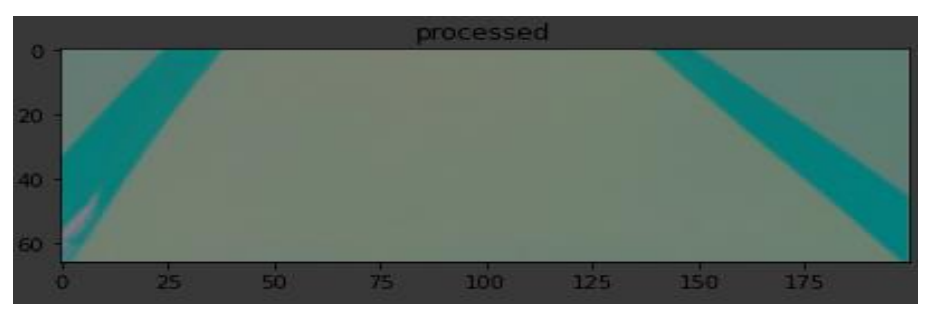

Fig.10: YUV Road Image

\section{6) CNN Architecture}

Based on NVIDIA architecture the CNN algorithm is based on 4 fully connected layer and 5 convolution operation. The first 3 convolution operation is made by $5 \times 5$ kernel or filter and the last 3 convolution is made by $3 \times 3$ filter. The activation function used is ELU. For training the model 252.219 trainable parameter has been used based all those parameter are used to achieve the best result. 


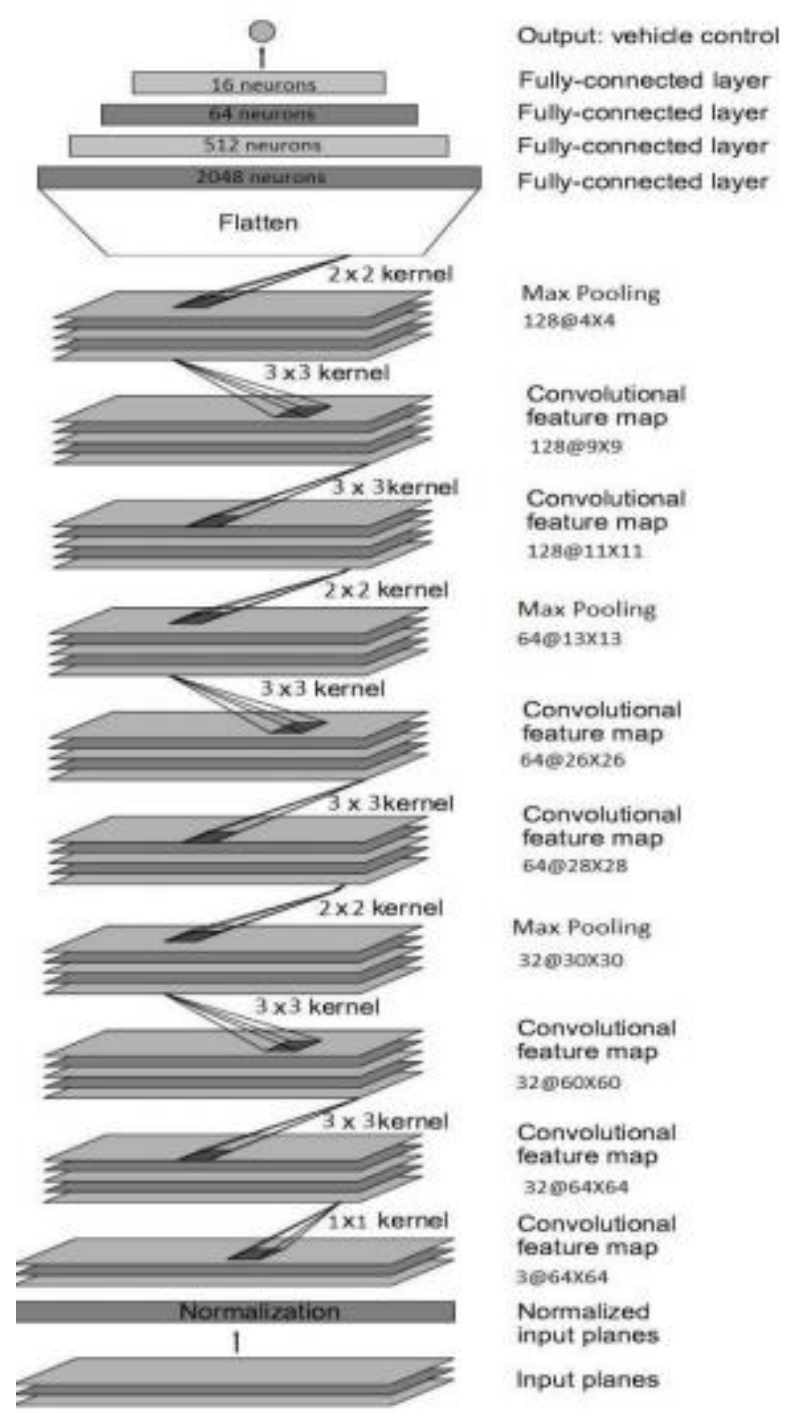

Fig 11: CNN Architecture.

\begin{tabular}{|c|c|c|}
\hline Layer (type) & Output Shape & Param \# \\
\hline conv2d_1 (Conv2D) & (None, $31,98,24$ ) & 1824 \\
\hline$\overline{\text { conv2d_2 (Conv2D) }}$ & (None, 14, 47, 36) & 21636 \\
\hline Conv2d_3 (Conv2D) & (None, 5, 22, 48) & 43248 \\
\hline conv2d_4 (Conv2D) & (None, 3, 20, 64) & 27712 \\
\hline dropout_1 (Dropout) & (None, $3,20,64$ ) & $\theta$ \\
\hline Conv2d_5 (Conv2D) & (None, $1,18,64$ ) & 36928 \\
\hline flatten_1 (Flatten) & (None, 1152) & $\theta$ \\
\hline dropout_2 (Dropout) & (None, 1152) & $\theta$ \\
\hline dense_1 (Dense) & (None, 100) & 115300 \\
\hline dense_2 (Dense) & (None, 50) & 5050 \\
\hline dense_3 (Dense) & (None, 10) & 510 \\
\hline dense_4 (Dense) & (None, 1) & 11 \\
\hline
\end{tabular}

Fig 12: Total number of parameters for training.

\section{RESULT}

Table 1: Convolution operation result after 10 epoch.

\begin{tabular}{|l|l|l|l|}
\hline $\begin{array}{l}\text { Convolutio } \\
\mathrm{n} \text { per epoch }\end{array}$ & $\begin{array}{l}\text { Epoch } \\
\text { phase }\end{array}$ & $\begin{array}{l}\text { Training } \\
\text { loss }\end{array}$ & $\begin{array}{l}\text { Validatio } \\
\mathrm{n} \text { loss }\end{array}$ \\
\hline 300 & $10 / 10$ & 115.0076 & 58.7779 \\
\hline
\end{tabular}

After the training process of the 560 images where 452 image was used for data training and the rest used for data validation. Based on the above results it seen that the training and validation loss rate has dropped instantly after the $4^{\text {th }}$ epoch. It also show that at each epoch phase 300 convolution operation is performed on the dataset as a result at $10^{\text {th }}$ epoch phase it shows a significant loss rate drop. The loss function used is Mean Square Error (MSE) because of the regression operation.

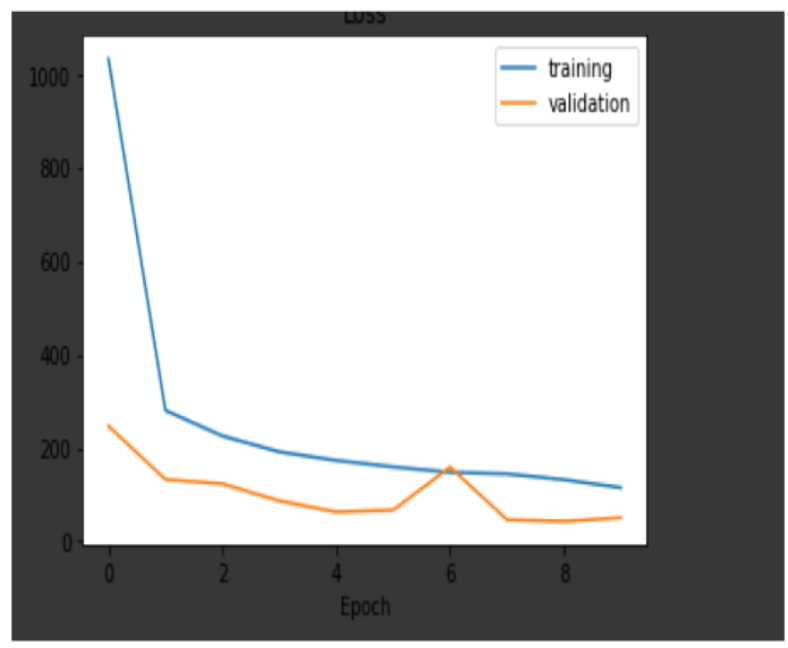

Fig 14: Training v/s Validation Loss.

No overfitting problem occurred during the convolution process as shown in graph. As the number of epoch increases the loss factor decreases as well so that means more number of epoch leads to a more accurate model. By using the ELU activation function it could generate negative outputs, which helps the network weights and biases in the desired 
direction. After the CNN operation we got $95 \%$ accuracy and about 0.5 error rate.

\section{VII.CONCLUSION}

There are different techniques with their own advantages and disadvantages to work in autonomous car computation. The capital aim of this analysis assignment is to propose techniques that enhance the operation and techniques for a better steering angle prediction. However for obtaining a better result the set of frames should be well processed or enhanced. During the convolution process we may face the overfitting problem. To overcome such problem we have added some dropout function. CNN can be effectively used for prediction of steering angle based on the direction of the road. The obtained rate of the experience results generated using actual driving images is $95 \%$ using 10 epoch for the evaluation.

\section{REFERENCES}

[1]. Oussama A, Mohamed T. A literature review of steering angle prediction algorithms for Selfdriving cars. :10.

[2]. Kluge K. Extracting road curvature and orientation from image edge points without perceptual grouping into features. Intell Veh Symp Proc. 1994;(3 13):109-114. doi:10.1109/ivs.1994.639482

[3]. Yuan C, Chen H, Liu J, Zhu D, Xu Y. Robust lane detection for complicated road environment based on normal map. IEEE Access. 2018;6:49679-49689. doi:10.1109/ACCESS.2018.2868976

[4]. Du S, Guo H, Simpson A. Self-Driving Car Steering Angle Prediction Based on Image Recognition. Cs231NStanfordEdu. 2017. http://cs231n.stanford.edu/reports/2017/pdfs/626. pdf.
[5]. Detec L, Luong QT. Primary Examiner Andrew W . Johns. 2004;1(12).

[6]. Schneiderman H, Nashman M. A Discriminating Feature Tracker for Vision-Based Autonomous Driving. IEEE Trans Robot Autom. 1994;10(6):769-775. doi:10.1109/70.338531

[7]. Mustafa R, Min Y, Zhu D. Obscenity detection using haar-like features and gentle Adaboost classifier. Sci World J. 2014;2014. doi:10.1155/2014/753860

[8]. nashman_m_1993_1.pdf.

[9]. Niu J, Lu J, Xu M, Lv P, Zhao X. Robust Lane Detection using Two-stage Feature Extraction with Curve Fitting. Pattern Recognit. 2016;59:225-233.

doi:10.1016/j.patcog.2015.12.010

[10].Veit T, Tarel JP, Nicolle P, Charbonnier P. Evaluation of road marking feature extraction. IEEE Conf Intell Transp Syst Proceedings, ITSC. 2008;(November):174-181. doi:10.1109/ITSC.2008.4732564

[11].Wang J, An X. A multi-step curved lane detection algorithm, based on hyperbola-pair model. 2010 IEEE Int Conf Autom Logist ICAL 2010.

2010:132-137. doi:10.1109/ICAL.2010.5585398

[12].Valiente R, Zaman M, Ozer S, Fallah YP. Controlling steering angle for cooperative selfdriving vehicles utilizing CNN and LSTM-based deep networks. IEEE Intell Veh Symp Proc. 2019;2019-June(April):2423-2428. doi:10.1109/IVS.2019.8814260

[13].Wang D, Wen J, Wang Y, Huang X, Pei F. Endto-End Self-Driving Using Deep Neural Networks with Multi-auxiliary Tasks. Automot Innov. 2019;2(2):127-136. doi:10.1007/s42154019-00057-1

[14].Chen J, Ruan Y, Chen Q. A precise information extraction algorithm for lane lines. China Commun. 2018;15(10):210-219. doi:10.1109/CC.2018.8485482 
[15].Jang HJ, Baek SH, Park SY. Curved lane detection using robust feature extraction. 2014 2nd Int Conf Syst Informatics, ICSAI 2014. 2015;(Icsai):109-112. doi:10.1109/ICSAI.2014.7009269

\section{Cite this article as :}

Aires Da Conceicao, Dr. Sheshang Degadwala, "Steering Angle Prediction Based on Road Direction using Convolution Neural Network (CNN)", International Journal of Scientific Research in Science and Technology (IJSRST), Online ISSN : 2395-602X, Print ISSN : 2395-6011, Volume 7 Issue 4, pp. 88-95, July-August 2020. Available at doi : https://doi.org/10.32628/IJSRST207429

Journal URL : http://ijsrst.com/IJSRST207429 